CPT-91/P.2628

November 1991

\title{
On polynomial solutions of differential equations
}

\author{
A.Turbiner[ \\ CPT, CNRS-Luminy, Marseille, F-13288, FRANCE \\ (JMP, Nov.-Dec.92)
}

\begin{abstract}
A general method of obtaining linear differential equations having polynomial solutions is proposed. The method is based on an equivalence of the spectral problem for an element of the universal enveloping algebra of some Lie algebra in the "projectivized" representation possessing an invariant subspace and the spectral problem for a certain linear differential operator with variable coefficients. It is shown in general that polynomial solutions of partial differential equations occur; in the case of Lie superalgebras there are polynomial solutions of some matrix differential equations, quantum algebras give rise to polynomial solutions of finite-difference equations. Particularly, known classical orthogonal polynomials will appear when considering $S L(2, \mathbf{R})$ acting on
\end{abstract}

\footnotetext{
${ }^{1}$ On leave of absence from: Institute for Theoretical and Experimental Physics, Moscow 117259, RUSSIA

E-mail: TURBINER@CERNVM or TURBINER@VXCERN.CERN.CH
} 
$\mathbf{R P}_{\mathbf{1}}$. As examples, some polynomials connected to projectivized representations of $s l_{2}(\mathbf{R}), s l_{2}(\mathbf{R})_{q}, \operatorname{osp}(2,2)$ and $s o_{3}$ are briefly discussed. 
An importance of polynomial solutions of differential equations is undoubtable for applications. From one side, they usually lead to closed analytical expressions, allowing to build constructive models of concrete physical phenomena. From other side, they can be used as an entry for performing perturbative or iterative considerations. In the paper we present a general method for generating linear differential equations of various types having polynomial solutions. The main idea is connected to exploiting so called "projectivized" finite-dimensional representations in differential operators of Lie algebras.

Take some Lie algebra $g$.

Definition. Let us call a projectivized representation of the Lie algebra $g$ the representation in a form

$$
J^{\alpha}=a^{\alpha, \mu}(x) \partial_{\mu}+b^{\alpha}(x) \quad, \quad J^{\alpha} \in g
$$

$\alpha=1,2, \ldots, \operatorname{dimg}, x \in R^{n-1}, \mu=1,2, \ldots, n-1$ and $a^{\alpha, \mu}(x), b^{\alpha}(x)$ are certain functions on $R^{n-1}$ and generically $b^{\alpha}(x)$ are non-vanishing simultaneously for all $\alpha^{\prime} s$.

One possible way to define such a representation (1) is the following. One can define a linear representation of $g$ in $\mathbf{R}^{n}$ (homomorphism $g \rightarrow g l(n, \mathbf{R})$ ). Then the expression (1) determines the corresponding action of $g$ in the projective space $\mathbf{R P}^{n-1}$ in affine coordinates. Hereafter, I will consider the restriction of the action of $g$ on $\mathbf{R}^{n-1} \subset \mathbf{R} \mathbf{P}^{n-1}$.

Let $g$ be a graded Lie algebra (for example, $g$ is graded, semi-simple). Then the universal enveloping algebra $U_{g}$ is endowed with the gradation corresponding to the gradation on $g$. Therefore one can give a natural definition of gradation of a monomial in universal enveloping algebra :

Definition. The gradation of a monomial in the generators is the sum of the gradations of multipliers.

Considering elements of a universal enveloping algebra $U_{g}$ (the algebra of all polynomials of the generators of $g$ ), one can define a regular element as follows

Definition. An element $h$ of universal enveloping algebra of a graded Lie algebra is called regular, if it contains at least one monomial in the 
generators with non-negative gradation.

It is worth emphasizing that a regular element $h \in U_{g}$ always contains a monomial, which can be represented by elements of the Cartan subalgebra only (see discussion e.g. [1]).

The generators (1) act on inhomogeneous functions (or sections of a certain bundle on $\mathbf{R}^{n-1}$ ). Let us consider a finite-dimensional representation $M^{d}$ of dimension $d$ of the Lie algebra $g$ in the form (1), which means the existence of an invariant finite-dimensional subspace $V^{d}$ in a certain functional space.

Lemma. If a Lie algebra $g$ has a finite-dimensional representation $M^{d}$ of dimension $d$, then any element $h \in U_{g}$ has a finite-dimensional representation of the same dimension $d$.

The representations in terms of differential operators of type (1) were introduced by S.Lie. However, they are poorly studied yet (history and last developments are given at [2]). In fact, any semi-simple Lie algebra can possess a projectivized representation (1).

Suppose that the invariant subspace of the finite-dimensional representation of $g$ in the form (1) can be parametrized by inhomogeneous polynomials in some coordinates.

Now, one formulates the main theorem.

Theorem. If a semi-simple Lie algebra g given by the representation (1) has an invariant subspace $V^{d}$ parametrized by inhomogeneous polynomials in some coordinates, for almost any polynomial element $h \in U_{g}$ the spectral problem

$$
h \varphi(x)=\varepsilon \varphi(x)
$$

possesses a certain number of eigenfunctions in the form of inhomogeneous, finite order polynomials. If $g$ is a graded algebra and $h$ is a regular element containing monomials of positive gradation, in general, the number of eigenfunctions in the form of a polynomial is equal to the dimension $d$ of an invariant subspace $V^{d}$. If a regular element $h$ has no terms with the positive gradation, an infinite sequence of eigenfunctions of (2) are represented by polynomials in some coordinates. 
This Theorem provides sufficient conditions for generating differential equations having, at least, one polynomial solution.

Now let us consider some concrete examples.

1. $g=s l_{2}(\mathbf{R})$.

A projectivized representation for the algebra $g=s l_{2}(\mathbf{R})$ has the form (see e.g. [3]) 2]

$$
\begin{gathered}
J^{+}=x^{2} \partial_{x}-2 j x, \\
J^{0}=x \partial_{x}-j, \\
J^{-}=\partial_{x},
\end{gathered}
$$

where $x \in \mathbf{R}^{1}$. Here the parameter $j$ is interpreted as the spin (or mark) of the representation. If $2 j$ is an non-negative integer, there will exist an invariant sub-space of dimension $(2 j+1)$ in the space of functions on $\mathbf{R}^{\mathbf{1}}$. The realization of this representation has a form of inhomogeneous polynomials in $x$

$$
R=\left\{1, x, x^{2}, \ldots, x^{2 j}\right\},
$$

Take, for instance, a polynomial element of $U_{s l_{2}(\mathbf{R})}$ in the form

$$
h_{s l(2, R)}=\sum_{n_{1}, n_{2}, n_{3} \geq 0}^{n_{1}+n_{2}+n_{3} \leq n} \mathcal{P}_{n_{1}, n_{2}, n_{3}}\left(J^{+}, J^{0}, J^{-}\right)
$$

of non-commutative variables $J^{+}, J^{0}, J^{-}$of degrees $n_{1}, n_{2}, n_{3}$, respectively. Let us fix some convention about ordering of generators in (5) to avoid the double counting due to commutation relations, e.g. in any monomial all $\mathrm{J}^{+}$ are situated at the left and $J^{-}$are placed at the right.

The algebra $g=s l_{2}(\mathbf{R})$ is graded and one can introduce the natural gradation of generators (3) as $+1,0,-1$, respectively. The terms with the positive gradation of (5) means an existence of monomials with $n_{1}>n_{3}$; zero gradation of (5) means an existence of terms with $n_{1}=n_{3}$ and/or $n_{2} \neq 0$ . Substituting (3) to (5) and then considering the spectral problem (2), one arrives at the spectral problem for the Fuchs-type operator

$$
P_{2 n}(x) \partial_{x}^{n} \varphi(x)+P_{2 n-1}(x) \partial_{x}^{n-1} \varphi(x)+\ldots+P_{n}(x) \varphi(x)=\varepsilon \varphi(x)
$$

\footnotetext{
${ }^{2}$ At the first time this representation has been described by S.Lie
} 
where $P_{k}$ are polynomials with coefficients related to the coefficients of the element (5). As a consequence of main Theorem, the spectral problem (6) can have up to $(2 j+1)$ solutions in the form of multi-parametrical polynomials of degree $2 j$ in $x$. The number of parameters of these polynomial solutions is defined by the number of non-trivial free parameters in (5). f It is worth noting that the Pochhammer's equation having a polynomial solution (see e.g. [4], Part I, Ch.5 (22)) occurs in our scheme as a particular case (6) with polynomial (5) without terms of positive gradation.

For the particular case of the second-order polynomial element of $U_{s l_{2}(\mathbf{R})}$ ( $n=2$ in (5)), the general solution of (6) gives rise to the recently discovered quasi-exactly-solvable problems [3], since always one can transform the spectral problem (6) (using a change of variable, $x=x(z)$ and gauge transformation, $\varphi=\psi \exp (a)$, where $a$ is some real function ) to the SturmLiouville problem. All known one-dimensional quasi-exactly-solvable problems [3] can be obtained in such a way. In general, it is 8-parametric family of the Schroedinger operators. If one considers the second-order elements of the form

$$
h=c_{\alpha \beta} J^{\alpha} J^{\beta}+c_{\alpha} J^{\alpha} \quad, \alpha, \beta=+,-, 0
$$

(assuming summation over repeating indeces) without terms of positive gradation

$$
h_{s l_{2}(\mathbf{R})}=c_{00} J^{0} J^{0}+c_{0-} J^{0} J^{-}+c_{--} J^{-} J^{-}+c_{0} J^{0}+c_{-} J^{-},
$$

the equation (6) coincides to the hypergeometrical equation under the condition of existance of truncated solutions. Corresponding eigenvalues are

$$
\epsilon_{n}=-c_{00} n(n-2 j)+c_{0} n+\text { const }
$$

Let us emphasis that the number of free parameters is equal to 5, which coincides to mysterious Ince number of free parameters of hypergeometri-

\footnotetext{
${ }^{3}$ Counting the number of free parameters of $h$ one should take into account all possible relations between generators (1). For instance, the quadratic Casimir operator in representation (3) becomes the number

$$
J^{+} J^{-}-J^{0} J^{0}+J^{0}=-j(j+1),
$$

It reduces effectively the number of free parameters for the second order polynomial element of $U_{s l_{2} \mathbf{R}}$.
} 
cal equation having truncated solutions. So, one has natural Lie-algebraic interpretation of the Ince number.

In particular, if one takes (7) in the form

$$
h_{s l_{2}(\mathbf{R})}=J^{-} J^{-}-2 J^{0}
$$

the standard Hermite equation $y \prime \prime-2 x y \prime+\lambda y=0$ appears. The element

$$
h_{s l_{2}(\mathbf{R})}=J^{0} J^{-}-J^{0}+[(a+1)+j] J^{-}
$$

leads to the generalized Laguerre equation $x y \prime \prime+(a+1-x) y \prime+\lambda y=0$ with the associated Laguerre polynomials as solutions. In turn, the element

$$
h_{s l_{2}(\mathbf{R})}=-J^{0} J^{0}+J^{-} J^{-}-(2 j+1) J^{0}
$$

leads to the Legendre equation $\left(1-x^{2}\right) y \prime \prime-2 x y \prime+\lambda y=0$. And finally, taking

$$
h_{s l_{2}(\mathbf{R})}=-J^{0} J^{0}+J^{-} J^{-}-(2 j+1+a+b) J^{0}+(b-a) J^{-},
$$

or,

$$
h_{s l_{2}(\mathbf{R})}=-J^{0} J^{0}+J^{0} J^{-}-(2 j+1+a+b) J^{0}+(a+1+j) J^{-},
$$

one gets a symmetric form of the Jacobi equation $\left(1-x^{2}\right) y / \prime+[b-a-(a+b+$ $2) x] y \prime+\lambda y=0$, an asymmetric form $x(1-x) y \prime \prime+[1+a-(a+b+2) x] y \prime+\lambda y=0$ (see e.g. [5]), respectively.

Remark. Above consideration can be easily extended for the case of several real variables taking $g=s l_{2}(\mathbf{R}) \oplus s l_{2}(\mathbf{R}) \oplus \ldots \oplus s l_{2}(\mathbf{R})$ (d times). Particularly, if the second-order polynomial element of $U_{g}$ has the form

$$
\begin{gathered}
h_{s l_{2}(\mathbf{R}) \oplus s l_{2}(\mathbf{R}) \oplus \ldots \oplus s l_{2}(\mathbf{R})}=-\sum_{i, l}^{d}\left(J_{i}^{0} J_{j}^{0}-\delta_{i l} J_{i}^{0} J_{l}^{-}\right)- \\
\left(G+2 \sum_{i=1}^{d} j_{i}+1\right) \sum_{i=1}^{d} J_{i}^{0}+\sum_{i=1}^{d}\left(\gamma_{i}+j_{i}\right) J_{i}^{-},
\end{gathered}
$$


one obtains the spectral problem $H \Phi=\lambda \Phi$ for the $d$-dimensional differential operator

$$
H=\sum_{i, l=1}^{d}\left(x_{i} \delta_{i l}-x_{i} x_{l}\right) \partial_{x_{i} x_{l}}+\sum_{i=1}^{d}\left(\gamma_{i}-G x_{i}\right) \partial_{x_{i}}
$$

with the $d$-dimensional Lauricella polynomials

$$
\Phi=F_{A}\left[\begin{array}{cc}
M+G-1 ;-m_{1}, \ldots,-m_{d} & \\
\gamma_{1}, \ldots, \gamma_{d} & \left(x_{1, \ldots,}, x_{d}\right)
\end{array}\right]
$$

(for definitions see e.g. [6]) as solutions, where $m_{i}=2 j_{i}$ are non-negative integers, $M=\sum_{i=1}^{d} m_{i}$ and the $\gamma_{i}$ are real positive numbers. In fact, the operator $H$ maps polynomials of maximum order $m_{i}$ in $x_{i}$ into polynomials of the same type. Lauricella polynomials play an important role as the explicit analytic solutions of the eigenvalue problem for modified Laplace-Beltrami operator on $d$-sphere (for further details see [6]).

2. $g=\mathrm{SO}_{3}$.

The finite-dimensional projectivized representation of $g=s_{3}$ is given by formulae (see [7])

$$
\begin{gathered}
J^{1}=\partial_{y}+y^{2} \partial_{y}+x y \partial_{x}-j y \\
J^{2}=y \partial_{x}-x \partial_{y} \\
J^{3}=-\partial_{x}-x^{2} \partial_{x}-x y \partial_{y}+j x
\end{gathered}
$$

where $x, y$ are the real variables, the spin $j$ is the non-negative integer and the dimension of the invariant subspace is equal to $(1+j)(1+j / 2)$. Invariant sub-space can be parametrized by polynomials

$$
R=\left\{1, x, y, x^{2}, x y, y^{2}, \ldots, x^{j}, x^{j-1} y, \ldots, x y^{j-1}, y^{j}\right\}
$$

Taking the polynomial element of $U_{s o(3)}$ in the form (5) (replacing formally $\left.J^{+} \rightarrow J^{1}, J^{0} \rightarrow J^{2}, J^{-} \rightarrow J^{3}\right)$, one comes to the spectral problem

$$
\begin{gathered}
P_{2 n}(x) \partial_{x}^{n} \varphi(x, y)+P_{2 n-1,1}(x, y) \partial_{x}^{n-1} \partial_{y} \varphi(x, y)+ \\
\ldots+P_{2 n}(y) \partial_{y}^{n} \varphi(x, y)+\ldots+P_{n}(x, y) \varphi(x, y)=\varepsilon \varphi(x, y)
\end{gathered}
$$


which has, in general, $(1+j)(1+j / 2)$ polynomial eigenfunctions. The examples of the polynomial solutions for the case $n=2$ are given in [7]. It is worth emphasizing that, if $n=2$ in (5) and an element $h$ obeys the condition: $c_{\alpha \beta}=c_{\beta \alpha}$ and $c_{\alpha}=0$, where $\alpha, \beta=1,2,3$, the spectral problem (17) always can be reduced by a gauge transformation, $\varphi(x, y)=\psi(x, y) \exp (a(x, y))$ to the spectral problem for the Schroedinger-type operator in curved space [7, []. It gives rise to the three parametric family of two-dimensional exactlysolvable problems [7].

Now let us consider a case of a Lie super-algebra.

3. $g=\operatorname{sl}(2, \mathbf{R} \mid \mathbf{2}) \oplus \mathbf{R}^{1}$.

The generators of $g=\operatorname{sl}(2, \mathbf{R} \mid 2) \oplus \mathbf{R}^{1}$ were obtained in $[7]$ and have the form

$$
\begin{gathered}
J^{+}=x^{2} \partial_{x}-2 j x+x \theta \partial_{\theta}, \\
J^{0}=x \partial_{x}-j+\frac{1}{2} \theta \partial_{\theta}, \\
J^{-}=\partial_{x} \\
T=-j-\frac{1}{2} \theta \partial_{\theta}
\end{gathered}
$$

for bosonic (even) generators and

$$
Q=\left[\begin{array}{c}
Q_{1} \\
Q_{2}
\end{array}\right]=\left[\begin{array}{c}
\partial_{\theta} \\
x \partial_{\theta}
\end{array}\right], \bar{Q}=\left[\begin{array}{c}
\bar{Q}_{1} \\
\bar{Q}_{2}
\end{array}\right]=\left[\begin{array}{c}
x \theta \partial_{x}-2 j \theta \\
-\theta \partial_{x}
\end{array}\right],
$$

for fermionic (odd) generators, where $x$ is the real variable and $\theta$ is the grassmanian one. Inspection of the generators shows that, if $2 j$ is nonnegative integer the finite-dimensional representation exists. Corresponding invariant sub-space has the dimension $(4 j+1)$ and can be parametrazed as

$$
R_{j}=\left(x^{0}, x^{1}, \ldots, x^{2 j}, x^{0} \theta, x^{1} \theta, \ldots, x^{2 j-1} \theta\right)
$$

Substituting (18),(19) into a polynomial element of the universal enveloping algebra and considering the spectral problem (2), one gets polynomial solutions. 
One can exploit the parametrization of $\theta$ and $\partial_{\theta}$ by Pauli matrices $\sigma_{+}$ and $\sigma_{-}$. Assuming the action of the generators (18),(19) on two-component spinors, one gets the representation the fermionic generators in the form

$$
Q=\left[\begin{array}{c}
\sigma_{-} \\
x \sigma_{-}
\end{array}\right], \bar{Q}=\left[\begin{array}{c}
x \sigma_{+} \partial_{x}-2 j \sigma_{+} \\
-\sigma_{-} \partial_{x}
\end{array}\right] .
$$

The representation (21) implies that in the spectral problem (2) an eigenfunction $\varphi(x)$ is treated as two-component spinor

$$
\varphi(x)=\left[\begin{array}{l}
\varphi_{1}(x) \\
\varphi_{2}(x)
\end{array}\right]
$$

and, finally, we come to the spectral problem for $2 \times 2$ matrix differential operator containing in general $(4 j+1)$ polynomial solutions.

The example of the spectral problem having polynomial eigenfunctions is given in [7]. Also in [7] the corresponding quasi-exactly-solvable spectral problem for the matrix Schroedinger operator is shown.

4. $g=s l_{2}(\mathbf{R})_{q}$.

Using quantum algebras, one can generate some finite-difference equations having polynomial solutions.

Let us build a $q$-analogue of the projectivized representation (3) [9]

$$
\begin{gathered}
T^{+}=x^{2} D-\{2 j\} x \\
T^{0}=x D-\hat{j} \\
T^{-}=D
\end{gathered}
$$

where $x$ is real variable, $\hat{j} \equiv \frac{\{2 j\}\{2 j+1\}}{\{4 j+2\}},\{n\}=\frac{1-p^{n}}{1-p}$ is the so-called quantum symbol and $p$ is a number characterizing the deformation; $D z=1+p z D$ and $D f(z)=\frac{f(z)-f(p z)}{(1-p) z}+f(p z) D$ is a shift or a finite-difference operator (or so called Jackson's symbol (see [11])). The generators (23) obey the commutation relations for a quantum algebra $g=s l_{2}(\mathbf{R})_{q}$ corresponding to so called the second Witten's deformation in a classification of C.Zachos [10] (for details, see [9]). In the limit $p \rightarrow 1$, (23) coincide to (3). 
A striking property of (23) consists of an existance of finite-dimensional representations. If $2 j$ is non-negative integer, generators (23) form a finitedimensional representation of dimension $2 j+1$ as well as for non-deformed case, $p=1$. Corresponding invariant sub-space can be presented by polynomials in $x$ of the order not higher than $2 j$ coinciding to (4).

One can build the universal enveloping algebra of $s l_{2}(\mathbf{R})_{q}$ in the same way as for ordinary Lie algebras (considering all possible polynomials in generators). As well as for non-deformed case, one can introduce a notation of gradation. The Theorem holds for this case as well. Taking the element $h_{g} \in U_{g}$ in the form (5), one comes to the spectral problem (2) for $n$-th order finite-difference operator $\mathrm{t}$ :

$$
P_{2 n}(x, p) D_{x}^{n} \varphi(x)+P_{2 n-1}(x, p) D_{x}^{n-1} \varphi(x)+\ldots+P_{n}(x, p) \varphi(x)=\varepsilon \varphi(x)
$$

(cf.(6)). Generically, the spectral problem (24) has $(2 j+1)$ polynomial solutions under the conditions of the main Theorem. In order to reproduce the known $q$-deformed classical Hermite, Laguerre, Legendre and Jacobi polynomials (for the latter, there exists the $q$-deformation of the asymmetric form (13) only) (see e.g. [11]), one should consider the spectral problem (2) 0 for the following combinations in the generators (23):

$$
\begin{gathered}
h_{s l(2, \mathbf{R})_{q}}=T^{-} T^{-}-\{2\} T^{0}, \\
h_{s l(2, \mathbf{R})_{q}}=T^{0} T^{-}-p^{-a-1} T^{0}+\left(p^{-a-1}\{a+1\}+\hat{j}\right) T^{-}, \\
h_{s l(2, \mathbf{R})_{q}}=-p T^{0} T^{0}+T^{-} T^{-}-[(2 \hat{j}-1) p+\{2\}] T^{0}, \\
h_{s l(2, \mathbf{R})_{q}}=-p^{a+b-1} T^{0} T^{0}+p^{a} T^{0} T^{-}- \\
{\left[(2 \hat{j}-1) p^{a+b-1}+\{a\} p^{b}+\{b\}\right] T^{0}+\left(\{a\}+\hat{j} p^{a}\right) T^{-},}
\end{gathered}
$$

respectively.

\footnotetext{
${ }^{4}$ The $q$-analogue of the spectral problem (2) is formulated as

$$
h \varphi(x)=\varepsilon \varphi(p x)
$$

in a little bit different way as usual(see e.g. 111). Inserting factor $p$ in r.h.s. gives no infuence on our consideration. Therefore we neglect this curcumstance.

${ }^{5}$ We consider corresponding difference equations following the book by Exton [1] : the equations (5.6.2), (5.5.7.1), (5.7.2.1), (5.8.3), respectively.
} 
Due to the fact, that representations (3) and (23) for the same spins have the same invariant functional sub-space (4), one can mix generators (3) and (23), and construct polynomials in generators containing both (3) and (23) as non-commutative variables. Evidently, those polynomials possess an invariant sub-space and in general corresponding spectral problem has polynomial solutions. It gives rise to the linear differential-difference equations in one variable having polynomial solutions.

\section{Acknowledgement}

In closing, I am very grateful to V.I.Arnold for numerous fruitful discussions and the interest to the subject. I would like to thank of C.S.Christ, C.Duval and V.Yu.Ovsienko for the useful discussions and careful reading of the manuscript and the Centre de Physique Theorique for the hospitality extended to me, where this work has been done. Also I thank to W. Miller, Jr. for suggestion to consider the Lauricella polynomials from present Liealgebraic point of view. 


\section{References}

[1] "Lie algabras"

N. Jacobson, Interscience Publ.Inc., New York, 1962

[2] "Quasi-exactly-solvable Lie algebras of differential operators in two complex variables"

A. Gonzales-Lopez, N. Kamran and P.J. Olver. Preprint of the School of Math., Univ. Minnesota 2/23/91 (1991)

[3] "Spectral Riemannian surfaces of the Sturm-Liouville operators and quasi-exactly-solvable problems"

A.V.Turbiner, Sov.Math.-Funk.Analysis i ego Prilogenia, 22 (1988) 9294 ;

"Quasi-exactly-solvable problems and $s l(2, R)$ algebra"

A.V. Turbiner, Comm. Math. Phys. 118 (1988) 467-474

[4] "Differentialgleichungen (losungsmethoden und losungen), I"

von Dr. E.Kamke, 6, Verbesserte Auflage, Leipzig, 1959

[5] "Ordinary differential equations and their solutions"

G.M. Murphy, van Nostrand, New York, 1960

[6] "Families of orthogonal and biorthogonal polynomials on the $N$-sphere" E.G. Kalnins, W. Miller, Jr. and M.V. Tratnik, SIAM J. Math. Anal. 22 (1991) 272-294

[7] "Quantal problems with partial algebraization of the spectrum" M.A. Shifman and A.V. Turbiner, Comm. Math. Phys. 126 (1989) 347-365

[8] "Quasi-exactly-solvable problems: one-dimensional analogue of rational conformal field theories"

A.Yu.Morozov, A.M.Perelomov, A.A.Rosly, M.A. Shifman and A.V. Turbiner,Int.Journ.Mod. Phys. A5 (1990) 803-843

[9] "sl $(2, \mathbf{R})_{q}$ and quasi-exactly-solvable problems"

O. Ogievetski and A. Turbiner, Preprint CERN-TH: 6212/91 (1991) 
[10] "Elementary paradigms of quantum algebras"

C. Zachos, Proceedings of the Conference on Deformation Theory of Algebras and Quantization with Applications to Physics, Contemporary Mathematics, J. Stasheff and M.Gerstenhaber (eds.), AMS, in press (1991)

[11] " $q$-Hypergeometric functions and applications" H. Exton, Horwood Publishers, 1983 